\title{
Matching heavy-light currents with NRQCD and HISQ quarks
}

\section{Christopher Monahan ${ }^{* a}$, Christine Davies $^{b}$, Ron Horgan ${ }^{c}$, G. Peter Lepage ${ }^{d}$, Heechang $\mathrm{Na}^{e}$ and Junko Shigemitsu ${ }^{f}$}

${ }^{a}$ Department of Physics, College of William and Mary, Williamsburg, VA 23187, USA

${ }^{b}$ SUPA, School of Physics \& Astronomy, University of Glasgow, Glasgow, G12 8QQ, UK

${ }^{c}$ DAMTP, Cambridge University, Cambridge, CB3 OWA, UK

${ }^{d}$ LEPP, Cornell University, Ithaca, NY 14853, USA

${ }^{e}$ Argonne National Laboratory, Argonne, IL 60439, USA

${ }^{f}$ Physics Department, The Ohio State University, Columbus, OH 43210, USA

\section{HPQCD Collaboration}

Email: c jmonahan@wm.edu

We calculate the one loop renormalisation parameters for the heavy-light axial-vector and vector currents using lattice perturbation theory. We use NonRelativistic QCD (NRQCD) heavy quarks and the Highly Improved Staggered Quark (HISQ) action for the light quarks. We present results for heavy-light currents with massless HISQ quarks and briefly discuss the extension to heavyheavy currents with massive HISQ quarks.

The 30th International Symposium on Lattice Field Theory

June 24-29, 2012

Cairns, Australia

${ }^{*}$ Speaker. 


\section{Introduction}

Recent tests of the unitarity of the Cabibbo-Kobayashi-Maskawa (CKM) matrix have indicated some tensions at the 2-3 $\sigma$ level [1-3]. In many cases, the constraints on CKM unitarity are limited by the precision of the theoretical inputs, in particular the hadronic matrix elements that characterise the strong interaction dynamics of weak processes. It is therefore imperative that these matrix elements are determined as precisely as possible.

The HPQCD collaboration is currently undertaking a suite of precision lattice calculations of heavy-light mesons to reduce the uncertainties associated with the theoretical inputs into CKM unitarity fits. New calculations of the decay constants $f_{B}$ and $f_{B_{s}}$ using the Highly Improved Staggered Quark (HISQ) action reached a precision at the $2 \%$ level $[4,5]$. These results are the most precise currently available for these decay constants and were made possible by the chiral properties and reduced taste-breaking uncertainties of the HISQ action.

In [5], the heavy-light currents were computed using HISQ light quarks and nonrelativistic QCD (NRQCD) heavy quarks. This calculation requires matching the heavy-light axial-vector and vector currents in the effective theory on the lattice with full QCD. In this article we report on the one loop perturbative matching of the HISQ-NRQCD currents for massless HISQ quarks. We also discuss the extension to heavy-heavy currents.

In the next section we describe the quark and gluon actions used in our calculation. We then review the matching formalism for heavy-light currents. In Section 4 we present our results for a range of heavy quark masses and include the quark renormalisation parameters. We discuss the extension to heavy-heavy currents in Section 5 and conclude in Section 6.

\section{Lattice Actions}

We use the Symanzik improved gluon action with tree level coefficients [6], given by

$$
S_{G}=-\frac{\beta}{3 u_{0}^{4}} \sum_{x, \mu>v}\left[5 P_{\mu v}-\frac{1}{4 u_{0}^{2}}\left(R_{\mu v}+R_{v \mu}\right)\right] .
$$

Here $P_{\mu v}$ is the usual plaquette and $R_{\mu v}$ the six-link loop, with $\beta=2 N_{c} / g^{2}$ and $u_{0}$ a tadpole improvement factor [7]. Radiative improvements to the gluon action do not contribute to our one loop matching calculation, because one loop radiative improvement generates an $\mathscr{O}\left(\alpha_{s}\right)$ insertion in the gluon propagator that would only contribute at higher orders.

We include a gauge-fixing term and, where possible, we confirm that gauge invariant quantities are independent of our choice of gauge parameter by working in both Feynman and Landau gauges, which we denote $\xi=1$ and $\xi=0$ respectively.

We use the Highly Improved Staggered Quark (HISQ) action [8] for the light quarks. The HISQ action has been used successfully to simulate both $b$ and $c$ quark systems $[9,10]$. Tastebreaking discretisation errors are significantly reduced by two levels of link fattening and $\mathscr{O}\left(a^{4} m_{0}^{4}\right)$ uncertainties are suppressed by powers of $v / c$ (where $v$ is the quark velocity) through a tuned a coefficient for the three-link "Naik" term [8]. We write the action as

$$
S_{\mathrm{HISQ}}=a^{4} \sum_{x} \bar{\psi}(x)\left(\gamma_{\mu} \nabla_{\mu}^{\mathrm{HISQ}}+m_{0}\right) \psi(x), \quad \text { with } \quad \nabla_{\mu}^{\mathrm{HISQ}}=\nabla_{\mu}^{(F U F)}-\frac{a^{2}}{6}(1+\varepsilon)\left(\nabla_{\mu}^{(U F)}\right)^{3} .
$$


The superscripts indicate that the first operator, $\nabla_{\mu}^{(F U F)}$, is built from the full HISQ-smeared links:

$$
\mathscr{F}_{\mu}^{\mathrm{HISQ}}=\left(\mathscr{F}_{\mu}-\sum_{\rho \neq \mu} \frac{a^{2}\left(\nabla_{\rho}\right)^{2}}{2}\right) \mathscr{U} \mathscr{F}_{\mu}, \quad \text { where } \quad \mathscr{F}_{\mu}=\prod_{\rho \neq \mu}\left(1+\frac{a^{2} \nabla_{\rho}^{(2)}}{4}\right)_{\text {symmetrised }}
$$

and $\mathscr{U}$ is a reunitarisation operator. The second operator in Equation (2.2), $\nabla_{\mu}^{(U F)}$, includes only one level of smearing. We work with massless quarks, so we set the bare quark mass, $m_{0}$, and the tuning parameter, $\varepsilon$, to zero.

For the heavy quark fields, $\Psi(x, t)$, we use the NRQCD action of [11], which is improved through $\mathscr{O}\left(1 / M_{0}^{2}\right)$ and $\mathscr{O}\left(a^{2}\right)$ and includes the leading relativistic $\mathscr{O}\left(1 / M_{0}^{3}\right)$ correction. The full NRQCD action is

$$
S_{\mathrm{NRQCD}}=\sum_{\mathbf{x}, t} \psi_{t}^{\dagger} \psi_{t-1}-\psi_{t}^{\dagger}\left(1-\frac{a \delta H}{2}\right)\left(1-\frac{a H_{0}}{2 n}\right)^{n} U_{4}^{\dagger}\left(1-\frac{a H_{0}}{2 n}\right)^{n}\left(1-\frac{a \delta H}{2}\right) \psi_{t-1}
$$

where $\psi_{t}^{\dagger}=\psi^{\dagger}(\mathbf{x}, t)$ and $\psi_{t-1}=\psi(\mathbf{x}, t-1)$. The leading kinetic term is given by

$$
H_{0}=-\frac{\Delta^{(2)}}{2 a M_{0}}
$$

and $\delta H$ includes higher order improvement terms, full details of which are given in, for example, [11]. We use the tree level values of $c_{i}=1$ for all the coefficients, $c_{i}$, of the higher order operators in $\delta H$, and do not consider the effects of radiative improvement of the NRQCD action.

\section{Matching Procedure}

On the lattice, the heavy-light axial-vector and vector current operators mix with higher order operators under renormalisation. We relate the lattice and continuum currents perturbatively and extract the mixing matrix elements at one loop. For massless HISQ quarks, the results for axialvector and vector currents are identical. Our strategy for the perturbative matching of heavy-light currents follows that developed in [12] and outlined in [13].

We require three lattice currents to match the temporal component of the vector current through $\mathscr{O}\left(\alpha_{s}, \Lambda_{\mathrm{QCD}} / M_{0}, \alpha_{s} /\left(a M_{0}\right), \alpha_{s} \Lambda_{\mathrm{QCD}} / M_{0}\right)$. These are

$$
J_{\mu}^{(0)}=\bar{q}(x) \Gamma_{\mu} Q(x), \quad J_{\mu}^{(1)}=-\frac{1}{2\left(a M_{0}\right)} \bar{q}(x) \Gamma_{\mu} \gamma \cdot \vec{\nabla} Q(x), \quad J_{\mu}^{(2)}=-\frac{1}{2\left(a M_{0}\right)} \bar{q}(x) \gamma \cdot \overleftarrow{\nabla} \gamma_{0} \Gamma_{\mu} Q(x)
$$

Here the $Q$ fields are four component Dirac spinors with the upper two components given by the two component NRQCD field and lower components equal to zero. The $\Gamma_{\mu}$ operator represents the vector current operator, $\Gamma_{\mu}=\gamma_{\mu}$.

The matrix elements of the vector current in full QCD are related to those in the effective theory via

$$
\begin{aligned}
& \left\langle V_{0}\right\rangle=\left(1+\alpha_{s} \rho_{0}^{\left(V_{0}\right)}\right)\left\langle J_{0}^{(0)}\right\rangle+\left(1+\alpha_{s} \rho_{1}^{\left(V_{0}\right)}\right)\left\langle J_{0}^{(1), \text { sub }}\right\rangle+\left(1+\alpha_{s} \rho_{2}^{\left(V_{0}\right)}\right)\left\langle J_{0}^{(2), \text { sub }}\right\rangle \\
& \left\langle V_{k}\right\rangle=\left(1+\alpha_{s} \rho_{0}^{\left(V_{k}\right)}\right)\left\langle J_{k}^{(0)}\right\rangle+\left\langle J_{k}^{(1), \text { sub }}\right\rangle .
\end{aligned}
$$


Here we have expressed the lattice currents in terms of the subtracted currents,

$$
J_{\mu}^{(i), \text { sub }}=J_{\mu}^{(i)}-\alpha_{s} \zeta_{i 0} J_{\mu}^{(0)},
$$

for $i=1,2$. The subtracted currents are more physical and have improved power law behaviour [12]. Note that, for the spatial components, we match through $\mathscr{O}\left(\alpha_{s}, \Lambda_{\mathrm{QCD}} / M_{0}, \alpha_{s} /\left(a M_{0}\right)\right)$.

We match at zero external quark momentum; the matching coefficients are given by

$$
\begin{aligned}
& \rho_{0}^{\left(V_{0}\right)}=\frac{1}{\pi}\left(\ln \left(a M_{0}\right)-\frac{1}{4}\right)-\frac{1}{2}\left(C_{q}+C_{H}\right)-\zeta_{00}^{\left(V_{0}\right)} \\
& \rho_{1}^{\left(V_{0}\right)}=\frac{1}{\pi}\left(\ln \left(a M_{0}\right)-\frac{19}{12}\right)-\frac{1}{2}\left(C_{q}+C_{H}\right)-C_{M}-\zeta_{01}^{\left(V_{0}\right)}-\zeta_{11}^{\left(V_{0}\right)} \\
& \rho_{2}^{\left(V_{0}\right)}=\frac{4}{\pi}-\zeta_{02}^{\left(V_{0}\right)}-\zeta_{12}^{\left(V_{0}\right)}, \\
& \rho_{0}^{\left(V_{k}\right)}=\frac{1}{\pi}\left(\ln \left(a M_{0}\right)-\frac{11}{12}\right)-\frac{1}{2}\left(C_{q}+C_{H}\right)-\zeta_{00}^{\left(V_{k}\right)}
\end{aligned}
$$

where the contributions from continuum QCD are given in $[12,13]$. The renormalisation parameters $C_{q}, C_{H}$ and $C_{M}$ are the one loop HISQ wavefunction renormalisation and the NRQCD wavefunction and mass renormalisation parameters respectively. We have written the pole mass, which is common to both lattice and continuum theories, in terms of the bare quark mass and must therefore include the one loop mass renormalisation in $\rho_{1}$.

The $\zeta_{i j}^{\left(V_{\mu}\right)}$ in Equations (3.5) to (3.8) are the one loop mixing matrix elements that arise from the mixing of the currents. The matrix element $\zeta_{02}$ includes a term that removes an $\mathscr{O}\left(a \alpha_{s}\right)$ discretisation error from $J_{0}^{(0)}[12,13]$. Thus our matching procedure ensures that $\mathscr{O}\left(\alpha_{s} / M_{0}\right)$ and $\mathscr{O}\left(a \alpha_{s}\right)$ corrections are made at the same time. There is a second dimension four current operator that is equivalent to $J_{0}^{(2)}$ via the equations of motion $[12,13]$, which we include in the determination of $\zeta_{i 2}($ for $i=1,2)$.

\section{Results}

We calculate the mixing matrix elements and renormalisation parameters with two independent methods. Our first method used the automated lattice perturbation theory routines HIPPY and HPSRC [14]. We performed these automated lattice perturbation theory calculations on the Darwin cluster at the Cambridge High Performance Computing Service and the Sporades cluster at the College of William and Mary with routines adapted for parallel computers using MPI (Message Passing Interface). Our second method used Mathematica and FORTRAN routines to construct the appropriate Feynman integrands; these were then evaluated with VEGAS [15].

We tested our code in a number of ways. We reproduced the NRQCD-AsqTad results of [13] and in many cases, we established that gauge invariant quantities, such as the mass renormalisation, were gauge parameter independent by working in both Feynman and Landau gauges. We also confirmed that our results exhibited the correct infrared behaviour, regulating any divergences with a gluon mass and using subtraction functions to ensure divergences were correctly handled by VEGAS. We believe that our two methods were sufficiently independent that agreement between these methods provides a stringent check of our results. 


\begin{tabular}{ccccccccc}
$a M_{0}$ & $C_{H}$ & $C_{M}$ & $\rho_{0}^{\left(V_{0}\right)}$ & $\rho_{1}^{\left(V_{0}\right)}$ & $\rho_{2}^{\left(V_{0}\right)}$ & $\zeta_{10}^{\left(V_{0}\right)}$ & $\rho_{0}^{\left(V_{k}\right)}$ & $\zeta_{10}^{\left(V_{k}\right)}$ \\
\hline 2.688 & $-0.360(2)$ & 0.262 & $-0.108(2)$ & $0.012(2)$ & $-0.712(4)$ & -0.1144 & $-0.034(2)$ & 0.0382 \\
2.650 & $-0.370(2)$ & 0.267 & $-0.111(2)$ & $0.013(2)$ & $-0.693(4)$ & -0.1157 & $-0.034(2)$ & 0.0386 \\
1.832 & $-0.658(2)$ & 0.466 & $-0.162(2)$ & $-0.042(3)$ & $-0.314(4)$ & -0.1593 & $0.018(2)$ & 0.0532 \\
1.826 & $-0.662(2)$ & 0.468 & $-0.164(2)$ & $-0.043(3)$ & $-0.311(4)$ & -0.1595 & $0.020(2)$ & 0.0532
\end{tabular}

Table 1: One-loop renormalisation parameters for the heavy-light vector current in Feynman gauge. For the $\rho_{i}^{\left(V_{\mu}\right)}$ the quoted uncertainties are the errors from each contribution added in quadrature, whilst for $C_{H}, C_{M}$ and the $\zeta_{10}^{\left(V_{\mu}\right)}$ the uncertainty is the statistical error from numerical integration. Unless otherwise indicated, the uncertainties are 1 or smaller in the final digit.

Many of the one loop parameters that we calculate are infrared divergent. We decompose our results into into an infrared finite term and an infrared divergent contribution, which we denote with a superscript ${ }^{\mathrm{IR}}$. Thus we write

$$
\begin{aligned}
Z_{q} & =1+\alpha_{s}\left(C_{q}^{\mathrm{IR}}+C_{q}\right)+\mathscr{O}\left(\alpha_{s}^{2}\right), & & C_{q}^{\mathrm{IR}}=\frac{1}{3 \pi}[1+(\xi-1)] \log \left(a^{2} \lambda^{2}\right), \\
Z_{H} & =1+\alpha_{s}\left(C_{H}^{\mathrm{IR}}+C_{H}\right)+\mathscr{O}\left(\alpha_{s}^{2}\right), & C_{H}^{\mathrm{IR}} & =\frac{1}{3 \pi}[-2+(\xi-1)] \log \left(a^{2} \lambda^{2}\right), \\
\widetilde{\zeta}_{00} & =\zeta_{00}+\zeta_{00}^{\mathrm{IR}}+\mathscr{O}\left(\alpha_{s}^{2}\right), & \zeta_{00}^{\mathrm{IR}} & =\frac{1}{3 \pi} \log \left(a^{2} \lambda^{2}\right) .
\end{aligned}
$$

Here $\lambda$ is the gluon mass, introduced to regulate the infrared behaviour, and $\xi$ is the gauge parameter. We confirm that both the gluon mass dependence and all infrared divergences cancel between the lattice and continuum one loop coefficients. Note that the NRQCD mass renormalisation, $Z_{M}=1+\alpha_{s} C_{M}+\mathscr{O}\left(\alpha_{s}^{2}\right)$, is infrared finite.

We tabulate results for the infrared finite contributions to the renormalisation parameters, mixing matrix elements and matching parameters for the heavy-light vector current at four different heavy quark masses in Table 4. For the NRQCD action we present results with $c_{i}=1$ and stability parameter $n=4$. We use the Landau link definition of the tadpole improvement factor, $u_{0}^{(1)}=0.7503(1)$. Only the matching coefficient $\rho_{1}$ has a tadpole correction coefficient. This correction contributes to $\zeta_{11}^{\left(A_{0}\right)}$ and is given by $\zeta_{11}^{u_{0}}=u_{0}^{(1)}$. The HISQ wavefunction renormalisation is of course independent of the NRQCD mass and we find $C_{q}(\xi=0)=0.1145(1)$ and $C_{q}(\xi=1)=-0.3940(1)$.

\section{Heavy-heavy Currents}

We are currently extending our calculation to heavy-heavy currents, with non-zero HISQ mass. Moving from massless to massive relativistic quarks complicates the matching procedure. In the former case, quarks and antiquarks at zero spatial momentum are indistinguishable and consequently scattering and annihilation processes give identical results. In the massive case, however, we must distinguish between quarks and antiquarks. Massive HISQ quarks also complicate the numerical integration considerably. The chief difficulty arises for the $A_{0}$ and $V_{k}$ annihilation currents, 
which contain a Coulomb singularity that must be handled with a subtraction function. Moreover, in the automated perturbation theory routines, the pole in NRQCD propagator crosses the integration contour and we must introduce a triple contour to ensure the stability of numerical integration [16].

\section{Summary}

We have determined the one loop matching coefficients and renormalisation parameters required to match the axial-vector and vector currents on the lattice to full QCD. We used the massless HISQ action for the light quarks and NRQCD for the heavy quarks and match at zero external quark momentum.

These matching coefficients are important ingredients in the determination of heavy-light mesonic decays in lattice QCD studies [5]. Studies of the $B_{s}$ meson using the relativistic HISQ action for both $b$ and $s$ quarks have recently been carried out [4]. Such an approach has the advantage that perturbative matching, which is generally the dominant source of error in the extraction of decay constants, is not required. Computations at the physical $b$ quark mass are currently prohibitively expensive, however, and require an extrapolation up to the $b$ quark mass. Furthermore, simulations of the $B$ meson are not yet feasible, because the use of light valence quarks and closeto-physical $b$ quark masses require large lattices with fine lattice spacings. Thus an effective theory approach to heavy-light systems remains the most efficient framework for precise predictions of $f_{B_{S}} / f_{B}$ and $f_{B}$. Such calculations require the perturbative matching calculation reported in this article.

The extension to heavy-heavy currents will enable the extraction of form factors for the $B \rightarrow$ $D^{(*)} \ell v$ semileptonic decays and the leptonic decay of the $B_{c}$ meson from lattice NRQCD computations. These calculations are underway.

\section{Acknowledgments}

The authors would like to thank Georg von Hippel for many helpful discussions. This work was supported by the DOE, the NSF and the STFC. Some of the computing was undertaken on the Darwin supercomputer at the HPCS, University of Cambridge, as part of the DiRAC facility jointly funded by the STFC.

\section{References}

[1] E. Lunghi and A. Soni, Demise of CKM and its aftermath, (2011), [1104.2117] ; J. Laiho, E. Lunghi and R.S. Van de Water, Flavor physics in the LHC era: the role of the lattice, PoS LAT2011 (2011), [1204.0791]

[2] J. Charles et al. (CKMfitter), Eur. Phys. J. C 41, 1 (2005), [hep-ph/ 0406184 ], updated results and plots available at: ckmfitter.in2p3.fr

[3] (UTfit) utfit.org

[4] C. McNeile et al. (HPQCD), High-precision $f_{B_{s}}$ and HQET from relativistic lattice QCD, Phys. Rev. D 85, 031503 (2012), [1110.4510] 
[5] H. Na et al. (HPQCD), The B and $B_{s}$ decay constants from lattice QCD, Phys. Rev. D 86, 034506 (2012), [1202.4914]

[6] M. Lüscher and P. Weisz, On-shell improved lattice gauge theories, Com. Math. Phys. 97, 59 (1985)

[7] G.P. Lepage and P.B. Mackenzie, Viability of lattice perturbation theory, Phys. Rev. D 48, 2250 (1993)

[8] E. Follana et al. (HPQCD), Highly improved staggered quarks on the lattice with applications to charm physics, Phys. Rev. D 75, 054502 (2007), [hep-lat/ 0610092 ]

[9] C. McNeile et al. (HPQCD), High-precision c and b masses, and QCD coupling from current-current correlators in lattice and continuum QCD, Phys. Rev. D 82, 034512 (2010), [1 004.4285 ]

[10] E.B. Gregory et al. (HPQCD), Precise $B, B_{s}$ and $B_{c}$ meson spectroscopy from full lattice QCD, Phys. Rev. D 83, 014506 (2011), [1010.3848] ; G.C. Donald et al. (HPQCD), Precision tests of the $J / \psi$ from full lattice QCD: mass, leptonic width and radiative decay rate to $\eta_{c},(2012)$, [1208.2855]; C. McNeile et al. (HPQCD), Heavy meson masses and decay constants from relativistic heavy quarks in full lattice QCD, Phys. Rev. D 86, 074503 (2012), [1207. 0994 ]

[11] A. Gray et al. (HPQCD), The Upsilon spectrum and $m_{b}$ from full lattice QCD, Phys. Rev. D 72, 094507 (2005) [hep-lat/ 0507013 ] ; R.J. Dowdall et al. (HPQCD), The Upsilon spectrum and the determination of the lattice spacing from lattice QCD including charm quarks in the sea, Phys. Rev. D 85, 054509 (2012), [1110.6887]

[12] C.J. Morningstar and J. Shigemitsu, One-loop matching of lattice and continuum heavy-light axial vector currents using NRQCD, Phys. Rev. D 57. 6741 (1998), [hep-lat/9712016]; C.J. Morningstar and J. Shigemitsu, Perturbative matching of lattice and continuum heavy-light currents with NRQCD heavy quarks, Phys. Rev. D 59. 094504 (1999), [hep-lat/9810 047 ]

[13] E. Gulez, J. Shigemitsu and M. Wingate, One-loop matching of the heavy-light $A_{0}$ and $V_{0}$ currents with NRQCD heavy and improved naive light quarks, Phys. Rev. D 69, 074501 (2004), [hep-lat/0409140]

[14] A.G. Hart et al., Automatically generating Feynman rules for improved lattice field theories, J. Comput. Phys. 209, 340 (2005), [hep-lat/0411026] ; A.G. Hart et al., Automated generation of lattice QCD Feynman rules, Comput. Phys. Commun. 180, 2698 (2009), [0 904 . 0375] ;

T.C. Hammant et al., Radiative improvement of the lattice NRQCD action using the background field method and application to the hyperfine splitting of quarkonium states, Phys. Rev. Lett. 107, 112002 (2011), [1105.5309]

[15] G.P. Lepage An adaptive multi-dimensional integration program, CLNS 80-447 (1980)

[16] E.H. Müller, A.G. Hart and R.R. Horgan, Renormalization of heavy-light currents in moving NRQCD, Phys. Rev. D 83, 034501 (2011), [1011.1215] 